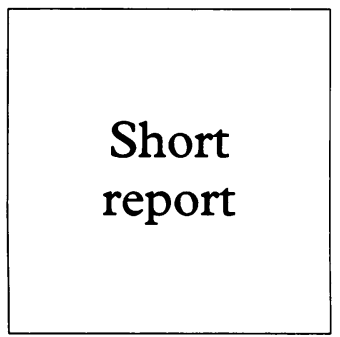

\title{
Family planning services in developing countries: an opportunity to treat asymptomatic and unrecognised genital tract infections?
}

David Wilkinson, Nomfanelo Ndovela, Abigail Harrison, Mark Lurie, Catherine Connolly, A Wim Sturm

Objective: To determine the prevalence of asymptomatic and unrecognised genital tract infections among women attending a family planning clinic in rural South Africa.

Methods: 189 consecutive women had genital samples taken to diagnose infection with Neisseria gonorrhoeae, Chlamydia trachomatis, Trichomonas vaginalis, Candida albicans, Treponema pallidum, and HIV, and to diagnose bacterial vaginosis.

Results: Mean age was 25 years; 155 (82\%) were unmarried, 156 (83\%) were currently using contraception, and $41(22 \%)$ reported having an STD treated in the preceding 12 months. Although none volunteered abnormal urogenital symptoms, 74 (39\%) had at least one elicited by direct questioning. 119 women $(63 \%)$ had at least one genital infection: $N$ gonorrhoeae (eight; $4 \%), C$ trachomatis $(14 ; 8 \%), T$ vaginalis $(26 ; 14 \%), C$ albicans $(56 ; 30 \%)$, active syphilis $(15$; $8 \%)$, HIV ( $44 ; 24 \%)$, and bacterial vaginosis $(29 ; 15 \%) .49$ women $(26 \%)$ had multiple infections. Most infections $(71 ; 60 \%)$ were asymptomatic. Symptomatic women failed to recognise and report their symptoms, and routine services failed to detect the infections.

Conclusion: Prevalence of genital tract infection is high among these women, most infections are asymptomatic, and symptomatic infections are frequently not recognised. Women attending family planning clinics in such settings should be screened for syphilis and offered testing for HIV infection. Strategies to detect and treat other genital infections need to be developed. (Genitourin Med 1997;73:558-560)

Keywords: family planning services; developing countries; genital tract infections

\section{Introduction}

Among women aged 15-44 years it is estimated that genital tract infections are the second most frequent cause of healthy life lost. ${ }^{1}$ Of the non-transmissible genital infections, candidiasis may be little more than a nuisance, but bacterial vaginosis is associated with low birth weight and premature labour. ${ }^{2}$ Sexually transmitted genital infections (STDs) are major causes of reproductive ill health, ${ }^{34}$ and they facilitate transmission of HIV. ${ }^{5}$

If STDs are to be controlled in developing countries, as well as providing syndromic management for symptomatic patients, it is crucial to identify and treat people with asymptomatic infections and those who have symptoms but do not recognise and report them. Family planning clinics may be important sites through which to identify, treat, and prevent such infections as many women in developing countries may attend them.

\section{Methods}

The study was done in the family planning clinic at Hlabisa hospital, South Africa. The area is rural and home to a Zulu speaking population. The clinic serves those living around Hlabisa village, a trading centre of about 8000 people. Approximately 120 women attend each month.

Women were informed about the study and offered a gynaecological examination. A questionnaire was administered and examination done by a female doctor. A high vaginal swab was used to make a smear for Gram staining and placed in Amies transport medium for culture of Trichomonas vaginalis. A cervical swab was used to make a smear for Chlamydia trachomatis direct immunoflourescence (DIF) (Microtrak, Syva) and was then placed in Amies transport medium for culture for Neisseria gonorrhoeae. Venous blood was taken for syphilis and HIV serology. Specimens were transported to the Department of Medical Microbiology, University of Natal Medical School, Durban and processed within 10 hours of sampling.

Diamond's medium, incubated at $37^{\circ} \mathrm{C}$ for a maximum of 7 days, was used to grow $T$ vaginalis. Growth was observed through wet mount microscopy done every day from day 2 on. The $C$ trachomatis DIF slide was prepared and read according to the manufacturer's instructions using fluorescence microscopy. $N$ gonorrhoeae culture was performed on New York City medium (Oxoid) and incubated for 48 hours at $37^{\circ} \mathrm{C}$ in a carbon dioxide incubator. Positivity was confirmed by the ability of isolated colonies to ferment glucose and not maltose, and by being oxidase positive. Bacterial vaginosis was diagnosed by microscopy of a Gram stained smear using Nugent's criteria. ${ }^{6}$ Presence of yeasts was established on the same Gram stained smear.

Active syphilis was defined as a positive RPR and a positive TPHA result. HIV infection was diagnosed when two different method 
ELISAs were positive. Women were offered HIV counselling and testing and in those that declined $(179 ; 95 \%)$ anonymous testing was done on spare serum with personal identifiers removed on completion of the study. Women were treated for infections and partners were referred for treatment. Ethical approval was granted by the ethics committee of the University of Natal Medical School.

Asymptomatic infection was defined as microbiologically diagnosed infection in the absence of any elicited urogenital symptoms (itch, discharge, dysuria, dyspareunia).

Unrecognised infection was defined as microbiologically diagnosed infection in the presence of any elicited urogenital symptoms, when these symptoms had not been volunteered by the patient.

Associations were assessed by means of the odds ratio and its $95 \%$ confidence interval. Differences between proportions were tested by the $\chi^{2}$ test. Continuous data were tested by ANOVA if normally distributed or the Kruskall-Wallis non-parametric test if not. Statistical significance was defined as a $\mathrm{p}$ value $<0.05$.

\section{Results}

Mean age was $25 \cdot 1$ (SD 6.0) years and 155 $(82 \%)$ were unmarried. Most $(156,83 \%)$ had attended the clinic previously and used injectable contraceptives (141, 90\%); others used an oral contraceptive $(14,9 \%)$ or condoms $(1,1 \%)$. Forty one women $(22 \%)$ reported having had an STD treated in the preceding 12 months.

No woman volunteered abnormal urogenital symptoms during her family planning consultation. When asked directly, the following symptoms were elicited from 74 women (39\%): genital itch (38; 20\%), vaginal discharge $(56 ; 30 \%)$, dysuria $(33 ; 18 \%)$, dyspareunia $(22 ; 12 \%)$, and genital ulcers $(4 ; 2 \%)$. Forty five women $(24 \%)$ had more than one symptom. None of age, marital status, current contraceptive use (or method), and history of

Table 1 Prevalence of genital tract infections, and the proportion that were asymptomatic, among 189 women attending a family planning clinic in rural South Africa

\begin{tabular}{lcc}
\hline Infection & $\begin{array}{l}\text { No (\%) } \\
\text { infected }\end{array}$ & $\begin{array}{l}\text { No (\%) } \\
\text { asymptomatic }\end{array}$ \\
\hline Chlamydia trachomatis & $14(8)$ & $5(36)$ \\
Neisseria gonorrhoeae & $8(4)$ & $3(37)$ \\
Trichomonas vaginalis & $26(14)$ & $15(58)$ \\
Candida albicans & $56(30)$ & $32(57)$ \\
Active syphilis & $15(8)$ & $10(67)$ \\
HIV infection & $44(24)$ & $23(52)$ \\
Bacterial vaginosis & $29(15)$ & $17(59)$ \\
Any GTI & $119(63)$ & $71(60)$ \\
\hline
\end{tabular}

*Active syphilis defined as RPR+/TPHA+.

Table 2 Relation between infection with Neisseria gonorrhoea, Chlamydia trachomatis, Trichomonas vaginalis, or bacterial vaginosis, and urogenital symptoms

\begin{tabular}{lllll}
\hline Symptom & $\begin{array}{l}\text { Odds ratio } \\
(95 \% \text { CI) }\end{array}$ & $p$ Value & Sensitivity & $\begin{array}{l}\text { Positive predictive } \\
\text { value }\end{array}$ \\
\hline Genital itch & $2 \cdot 2(1 \cdot 0-4 \cdot 9)$ & 0.05 & $29 \%$ & $50 \%$ \\
Vaginal discharge & $1 \cdot 8(0 \cdot 9-3 \cdot 6)$ & 0.09 & $38 \%$ & $45 \%$ \\
Dysuria & $1 \cdot 7(0 \cdot 7-3 \cdot 9)$ & 0.2 & $23 \%$ & $45 \%$ \\
Dyspareunia & $1 \cdot 7(0 \cdot 6-4 \cdot 4)$ & 0.3 & $15 \%$ & $45 \%$ \\
Any & $1 \cdot 6(0 \cdot 8-3 \cdot 2)$ & 0.1 & $47 \%$ & $42 \%$ \\
\hline
\end{tabular}

previous STD was significantly associated with any symptom. On examination, five women had vulval ulcers, four had vulvo-vaginal warts, and 95 (50\%) had an abnormal vaginal discharge (82 yellow/green in colour; 13 white and curd-like).

Most women $(119,63 \%)$ had at least one genital infection (table 1), and $49(26 \%)$ had multiple infections. A total of 80 women $(42 \%)$ had at least one STD. Most infections were asymptomatic (table 1 ). The rest, while symptomatic, were unrecognised or not reported. Furthermore, as syphilis screening was not done by the service, as HIV testing was not offered, and as no microbiological facilities were available, no infections were detected during the routine family planning consultation.

Further examining the relation between symptoms and infections we focused on $N$ gonorrhoeae, $C$ trachomatis, $T$ vaginalis, and bacterial vaginosis. Candidiasis was excluded as serious sequalae are uncommon, and syphilis and HIV infection were excluded because both can be reliably screened for serologically in the primary care setting. Of the 66 women with gonorrhoea, chlamydial infection, trichomoniasis, or bacterial vaginosis, 35 (53\%) were asymptomatic. Of the 31 with elicited symptoms, only five $(8 \%)$ had reported a gynaecological complaint when asked. The association between these four infections and elicited symptoms was weak (table 2). Sensitivity of symptoms for infection ranged from $15 \%$ to $47 \%$, and positive predictive value ranged from $42 \%$ to $50 \%$.

A genital ulcer was observed in six (3\%) of 189 women; five were vulval and one was cervical. Only two women were aware of the ulcer, and neither had reported it. None of these women had active syphilis.

\section{Discussion}

We have documented a high prevalence of genital tract infections and HIV infection among women attending the family planning clinic serving a rural South African population. Most infections were asymptomatic, the rest were symptomatic but unrecognised or unreported, and important opportunities to detect syphilis and HIV infection were missed by the service. Although family planning clinics offer a potentially important opportunity to detect and treat STDs and other genital infections, diagnosis in resource poor settings is difficult.

The high prevalence of asymptomatic infections is disturbing as diagnosis depends on a microbiological service that is rarely available in such settings. Detecting symptomatic infections may also be difficult if women either do not recognise abnormal symptoms or feel unable to report such symptoms in a family planning clinic. Elicited symptoms were weakly associated with infection and had poor positive predictive value, implying that a symptom based approach to management is ineffective in this setting. Further investigation to explore and understand women's percep- 
tions of their own health in this setting is warranted and this information could be used to develop health promotion messages.

By not screening for syphilis the routine family planning service failed to detect treatable infection in these women. Simple and accurate technology exists that enables this to be done on site. ${ }^{7}$ There is evidence that voluntary testing and counselling for HIV infection in developing countries may reduce $\mathrm{HIV}$ and STD transmission and increase condom use, ${ }^{89}$ but the low uptake in this study suggests that promoting such a service may be difficult.

If family planning clinics are to be part of efforts to control STDs in Africa, effective partner notification strategies will need to be developed, perhaps using index cases to actually treat partners. This may be very difficult if male partners do not support attendance of their female partners at family planning clinics. Contraceptive methods that women can use and control, and that provide protection against STDs and HIV, such as vaginal micro- bicides and the female condom, could also contribute to disease control.

1 World Bank. World Development Report, 1993. World Bank, Washington, 1993.

2 Hauth JC, Goldenberg RL, Andrews WW, DuBard MB, Copper RL. Reduced incidence of preterm delivery with metronidazole and erythromycin in women with bacterial vaginosis. N Engl f Med 1995;333:1732-6.

3 Schult KF, Cates W Jr, O'Mara PR. Pregnancy loss, infant death and suffering: legacy of syphilis and gonorinfant death and suffering: legacy of syphilis and

4 World Health Organisation. An overview of selected curWorld Health Organisation. An overview of selected cur-
able sexually transmitted diseases. WHO/GPA/STD 95.1. Geneva: World Health Organisation.

5 Grosskurth H, Mosha F, Todd J, Awijaruba E, Klokke A Senkoro K, et al. Impact of improved treatment of sexually transmitted diseases on HIV infection in rural Tanzania: randomised controlled trial. Lancet 1995;346:530-6.

6 Nugent RP, Krohn MA, Hillier SL. Reliability of diagnosis of bacterial vaginosis is improved by a standardised method of Gram stain interpretation. $f$ Clin Microbiol 1991;29:297-301.

7 Delport SD. On-site screening for maternal syphilis in an antenatal clinic. $S$ Afr Med F 1993;83:723-4.

8 Allen S, Tice J, Van de Perre P, Serufilira A, Hudes E, Nsengumuremyi $F$, et al. Effect of serotesting with counselling on condom use and seroconversion among HIV discordant couples in Africa. $B M F$ 1992;304:1605-9.

9 Allen S, Serufilira A, Bogaerts J, Van de Perre P, Nsengumuremyi F, Lindan C, et al. Confidential HIV testing and condom promotion in Africa. Impact on HIV and gonorrhoea rates. $\mathcal{F} A M A 1992 ; 268: 3338-43$. 